\title{
A Consensus-based Approach for Platooning with Inter-Vehicular Communications
}

\author{
S. Santini*, A. Salvi*, A. S. Valente*, A. Pescapè* \\ ${ }^{*}$ University of Napoli Federico II (Italy) \\ \{stefania.santini,alessandro.salvi\}@unina.it \\ \{antoniosaverio.valente,pescape $\} @$ unina.it
}

\author{
M. Segata ${ }^{\dagger \ddagger}$, R. Lo Cigno \\ †University of Innsbruck (Austria) \\ $\ddagger$ University of Trento (Italy) \\ \{locigno,msegata\}@disi.unitn.it
}

\begin{abstract}
Automated and coordinated vehicles' driving (platooning) is gaining more and more attention today and it represents a challenging scenario heavily relying on wireless Inter-Vehicular Communication (IVC). In this paper, we propose a novel controller for vehicle platooning based on consensus. Opposed to current approaches where the logical control topology is fixed a priori and the control law designed consequently, we design a system whose control topology can be reconfigured depending on the actual network status. Moreover, the controller does not require the vehicles to be radar equipped and automatically compensates outdated information caused by network delays. We define the control law and analyze it in both analytical and simulative way, showing its robustness in different network scenarios. We consider three different wireless network settings: uncorrelated Bernoullian losses, correlated losses using a Gilbert-Elliott channel, and a realistic traffic scenario with interferences caused by other vehicles. Finally, we compare our strategy with another state of the art controller. The results show the ability of the proposed approach to maintain a stable string of vehicles even in the presence of strong interference, delays, and fading conditions, providing higher comfort and safety for platoon drivers.
\end{abstract}

\section{INTRODUCTION}

The idea of automated and coordinated vehicles' driving goes back to the PATH project in California during the eighties' [1]. The main goals of the PATH project were freeing the driver from some driving chores, improving safety, and increasing road usage by reducing the vehicles' inter-distance. Since such goals were not achievable using standard sensor-based Adaptive Cruise Control (ACC), the community started to work on a different type of cruise control, named Cooperative Adaptive Cruise Control (CACC). What differentiates a CACC from a standard ACC is the use of wireless communications to share information such as speed and acceleration among vehicles, enabling the possibility to reduce inter-vehicle distance without compromising safety.

A group of coordinated vehicles is called a platoon. Building and managing a platoon requires multiple technologies. Essential to guarantee vehicles' coordination are: i) a control algorithm that regulates the relative distance with respect to the vehicle ahead and coordinate all vehicles to stabilize the platoon; and ii) a communication network to exchange information between vehicles. The control algorithm can use data received from multiple vehicles in the platoon, defining the control topology. As an example, the CACC designed in [2] considers data from the front vehicle only, while the one in [3] exploits data from the leader as well. What current approaches assume is a static control topology, which means that the design of the controller is based on a fixed communication pattern. When such communication pattern changes due to, for example, network impairments, the CACC is not able to safely control the platoon anymore.

In this paper, we overcome this problem by developing a flexible control system that can be reconfigured based on the actual communication capabilities. The contribution of this work is threefold. First, we develop a novel control algorithm based on a distributed consensus, with the goal of coordinating all vehicles to reach an equal inter-vehicle gap [4]. Our approach is specifically designed to take into account communication logical topology, as well as impairments as delay and losses. We provide the details of the control design, the control-loop dynamics, and the analysis of the stability of the proposed algorithm. Second, we implement the communication strategy to support the algorithm in Veins [5], and we carry out experiments with eight and sixteen cars in a realistic $10 \mathrm{~km}, 4$ lanes stretch of highway exploring different network-related impairments by including different packet loss models and by considering other cars equipped with InterVehicular Communication (IVC) capabilities interfering with the platoon. The communication delay, instead, is intrinsically modeled in Veins with a realistic communications device (IEEE $802.11 \mathrm{p}$ card) implementation. Third, we perform a comparison with a well known CACC algorithm [3] and show that our proposal is superior in terms of settling time (thus faster convergence) and damping of disturbances (thus providing higher comfort for platoon drivers). This paper extends the state of the art proposing, and proving the viability of, a control approach for vehicles platooning based on a consensus algorithm specifically designed to cope with IVC heterogeneous and time varying delays.

\section{Scenario, Motivation, AND Related Work}

We assume a standard Dedicated Short Range Communications / Wireless Access in the Vehicular Environment (DSRC/WAVE) [6], [7] access network with beaconing messages, and proper integration of different components of a cooperative driving system (emergency braking [8], anticollision techniques, etc.) that are not discussed in this work. 
The paper focuses on the algorithm and protocol necessary to form and stabilize a platoon, looking forward a robust technique that is tolerant to errors and impairments. The scenario and dynamic model are those described in [3].

Usually, in CACC strategies the controller parameters are tuned to attenuate the propagation of motion signals toward the tail of the platoon, i.e. to guarantee the so called string stable behavior once the platoon is engaged. Predecessor-following architectures based on pairwise interactions were shown to be highly sensitive to external disturbances and number of vehicles resulting into instabilities [9]. At the same time it is known that a speed dependent spacing policy, based on the headway time, leads to a string stable platoon for choices of the headway time consistent with the platooning application [10]. Control methods for ensuring platoon string stability exist under the assumption of the use of IVC without delays, i.e. the analytical stability analysis is carried out under the hypothesis of ideal communications [11]. This is not a realistic assumption, and communication delays are known to create hardly manageable string instability [12]. Recent research activities are addressed to design CACC strategies able to mitigate the effects of communication delays (see for example [13] and references therein). Since effects and limitations due to communication features are not explicitly accounted for during the control design, they are numerically investigated through sensitivity analysis, usually performed in presence of fixed, unique, and constant communication delay [12], [14]. Several approaches to evaluate the performance and stability of CACC strategies with respect to communication characteristics (as delay, packet losses, reliability, traffic and mobility dynamics) are based on proper simulation tools like [5]. As an example of this research direction, in [15] the performance of a CACC control algorithm (and its robustness with respect to periodic disturbances on the leader dynamics) are discussed in the presence of packet loss, network failures and beaconing frequencies. The simulation framework is built with a CACC controller prototype (designed in [16]), a traffic simulator (SUMO), and a network simulator (OMNeT++). The communication behavior (based on IEEE 802.11p) is modeled using $\mathrm{OMNeT++}$. Although platoon vehicles are in general modeled like a string, different control topologies may arise depending on the communication pattern among vehicles, and how the information is used by the control algorithm. If we consider generic control topologies, the problem of stabilizing a platoon naturally integrates in the more general framework of multi-agent systems control [17]. Further examples of this very recent research direction can be also found in [18], where a leaderless strategy is proposed for three autonomous vehicles ideally moving in a circle and sharing information across an allto-all configuration via IVC affected by a very simple constant and common delay. Platooning as a weighted and constrained consensus control problem is also discussed in [4], with the goal of understanding the influence of the control topology on the platooning dynamics by using a discrete-time Markov chain based approach, but without considering the effect of time-varying delays on the ensemble stability. Previous works in this field indeed consider IEEE 802.11-based radios as the technology to be employed in platooning systems [2], [19]. Phenomena like shadowing and fading, plus a highly concurrent channel access, can result in packet losses [20], [21] and thus highly variable and time dependent delays, possibly leading to instabilities in the control system. According to the literature and IVC standards, the frequency at which each vehicle has to broadcast its data must be no lower than $10 \mathrm{~Hz}$ [2], a value that imposes tight communication constraints, stress the channel load (the road and the safety communication channel are shared by all vehicles), but finds its justification in the dynamics of the vehicles, and thus can be considered a hard physical requirement.

Within this scenario, this paper tackles and solves the platoon control as a high order consensus problem accounting for time varying communication delays and vehicles dynamics. Detailed simulations in Veins, including realistic details such as vehicles' masses and inertia, actuation lags, packet losses and interfering traffic, show the performance of the proposed approach and its advantages. The idea is to find a proper decentralized control algorithm so that the emerging platoon topology, depending on the communication links, is asymptotically stable without the need of pre-establishing (with respect to the controller design) the topology. The main goal of the proposed approach is to guarantee the platoon stability in presence of heterogeneous and time-varying communication delays. This feature can be very important also in case of emergencies, when the control of vehicles must be returned to drivers, giving more time to perform this delicate action as the platoon remains stable for longer times even when the communication topology changes. The analysis of this possibility is however not carried out in this work. The control algorithm significantly enhances the theoretical analysis in [22] since it embeds velocity-dependent spacing policy and standstill requirements [16]. Furthermore, it overcomes the limitations of the stability analysis in [22] in which, for each vehicle, a unique aggregate delay (resulting from the fusion of different delays from different sources) was assumed.

\section{Platooning Control}

\section{A. Mathematical Preliminaries and Nomenclature}

The inter-vehicle communication structure can be modeled by a graph where every vehicle is a node. Hence, a platoon of $N$ vehicles is represented as a directed graph (digraph) $\mathcal{G}=$ $(\mathcal{V}, \mathcal{E}, \mathcal{A})$ of order $N$ characterized by the set of nodes $\mathcal{V}=$ $\{1, \ldots, N\}$ and the set of edges $\mathcal{E} \subseteq \mathcal{V} \times \mathcal{V}$. The topology of the graph is associated to an adjacency matrix with nonnegative elements $\mathcal{A}=\left[a_{i j}\right]_{N \times N}$. In what follows we assume $a_{i j}=1$ in the presence of a communication link from node $j$ to node $i$, otherwise $a_{i j}=0$. Moreover, $a_{i i}=0$ (i.e., self-edges $(i, i)$ are not allowed unless otherwise indicated). The presence of edge $(i, j) \in \mathcal{E}$ means that vehicle $i$ can obtain information from vehicle $j$, but not necessarily vice versa.

In the rest of the paper we consider $N$ vehicles together with a leader vehicle taken as an additional agent labelled with the index zero, i.e., node 0 . We use an augmented directed graph 
$\overline{\mathcal{G}}$ to model the platoon topology based on the communication pattern desired by the consensus algorithm, i.e., the existence of edge $(i, j)$ means that $i$ uses the information received by $j$ and not only that $i$ is within the communication range of $j$. We assume node 0 is globally reachable in $\overline{\mathcal{G}}$ if there is a path in $\overline{\mathcal{G}}$ from every node $i$ in $\mathcal{G}$ to node 0 [23].

Before proceeding to design our consensus controller, we recall here some useful results on the stability of delayed systems.

Let $C\left([-r, 0], \mathbb{R}^{n}\right)$ be a Banach space of continuous functions defined on an interval $[-r, 0]$ and taking values in $\mathbb{R}^{n}$ with a norm $\|\varphi\|_{c}=\max _{\theta \in[-r, 0]}\|\varphi(\theta)\|,\|\cdot\|$ being the Euclidean norm. Given a system of the form:

$$
\begin{gathered}
\dot{x}=f\left(x_{t}\right), t>0, \\
x(\theta)=\varphi(\theta), \theta \in[-r, 0],
\end{gathered}
$$

where $x_{t}(\theta)=x(t+\theta), \forall \theta \in[-r, 0]$ and $f(0)=0$, it holds:

Theorem 1. (Lyapunov-Razumikhin) [24]. Given system Eq. (1), suppose that the function $f: C\left([-r, 0], \mathbb{R}^{n}\right) \rightarrow \mathbb{R}^{n}$ maps bounded sets of $C\left([-r, 0], \mathbb{R}^{n}\right)$ into bounded sets of $\mathbb{R}^{n}$. Let $\psi_{1}, \psi_{2}$, and $\psi_{3}$ be continuous, nonnegative, nondecreasing functions with $\psi_{1}(s)>0, \psi_{2}(s)>0, \psi_{3}(s)>0$ for $s>0$ and $\psi_{1}(0)=\psi_{2}(0)=0$. If there is a continuous function $V(t, x)$ (Lyapunov-Razumikhin function) such that:

$$
\psi_{1}(\|x\|) \leq V(t, x) \leq \psi_{2}(\|x\|), t \in \mathbb{R}, x \in \mathbb{R}^{n},
$$

and there exists a continuous non decreasing function $\psi_{4}(s)$ with $\psi_{4}(s)>s, s>0$ such that :

$$
\begin{aligned}
\dot{V}(t, x) & \leq-\psi_{3}(\|x\|) \\
\text { when } V(t+\theta, x(t+\theta)) & <\psi_{4}(V(t, x(t))), \theta \in[-r, 0],
\end{aligned}
$$

then the solution $x=0$ is uniformly asymptotically stable.

\section{B. Consensus based Control Design}

The goal of the platoon control is to regulate speed and relative distance of each vehicle with respect to its predecessor and a leading vehicle respectively [11], [25]. Hence, a platoon is composed of a string of $N$ vehicles plus the additional leading vehicle acting as a reference for the ensemble. In our analysis each vehicle is equipped with on-board sensors to measure its absolute position, speed and acceleration, while an IEEE $802.11 \mathrm{p}$ radio enables vehicle to share information among neighbors and to receive leading vehicle reference signals.

The generic $i$-th vehicle dynamics can be described as the following inertial agent $(i=1, \ldots, N)$ :

$$
\begin{aligned}
& \dot{r}_{i}(t)=v_{i}(t) \\
& \dot{v}_{i}(t)=\frac{1}{M_{i}} u_{i}(t),
\end{aligned}
$$

where $r_{i}[\mathrm{~m}]$ and $v_{i}[\mathrm{~m} / \mathrm{s}]$ are the $i$-th vehicle absolute position (with respect to a given reference framework) and speed; $M_{i}$ $[\mathrm{kg}]$ is the $i$-th vehicle mass and the propelling force $u_{i}$ denotes the control input to be appropriately chosen to achieve the control goal. Similarly, the leader vehicle dynamics are

$$
\begin{aligned}
& \dot{r}_{0}(t)=v_{0} \\
& \dot{v}_{0}=0 .
\end{aligned}
$$

being $r_{0}$ and $v_{0}$ the leader state variables. Given Eqs. (4) and (5), the problem of maintaining a desired inter-vehicle spacing policy and a common speed can be rewritten as the following high-order consensus problem:

$$
\begin{aligned}
& r_{i}(t) \rightarrow \frac{1}{\Delta_{i}}\left\{\sum_{j=0}^{N} a_{i j} \cdot\left(r_{j}(t)+d_{i j}\right)\right\} \\
& v_{i}(t) \rightarrow v_{0} .
\end{aligned}
$$

where $d_{i j}$ is the desired distance between vehicles $i$ and $j ; a_{i j}$ (for $i=1, \ldots, N$ and $j=0, \ldots, N$ ) models the platoon topology emerging from the presence/absence of a communication link between vehicles $i$ and $j ; \Delta_{i}=\sum_{j=0}^{N} a_{i j}$ is the degree of vehicle/agent $i$, i.e., the number of vehicles establishing a communication link with vehicle $i$. Note that according to [26] the desired spacing $d_{i j}$ can be expressed as $d_{i j}=h_{i j} v_{0}+d_{i j}^{s t}$, where $h_{i j}$ is the constant time headway (i.e., the time necessary to vehicle $i$-th to travel the distance to its predecessor), and $d_{i j}^{s t}$ is the distance between the vehicles $i-$ th and $j-$ th at standstill. Furthermore we remark that $a_{i j}$ are the nonnegative elements of the adjacency matrix associated to the platoon topology directed graph $\overline{\mathcal{G}}$. In what follows we also assume that $a_{0 j}=0(\forall j=0, \ldots, N)$, since the leader does not consider data from any other vehicle.

The platoon high-order consensus problem in Eq. (6) is solved here by the following decentralized control action embedding the spacing policy information as well as all the time-varying communication delays:

$$
\begin{aligned}
& u_{i}=-b\left[v_{i}(t)-v_{0}\right]+ \\
& -\frac{1}{\Delta_{i}} \sum_{j=0}^{N} k_{i j} a_{i j}\left[r_{i}(t)-r_{j}\left(t-\tau_{i j}(t)\right)-\tau_{i j}(t) v_{0}-h_{i j} v_{0}-d_{i j}^{s t}\right]
\end{aligned}
$$

where $k_{i j}$ and $b$ are control gains to be opportunely tuned to regulate the mutual behavior among neighbor vehicles; $\tau_{i j}(t)$ and $\tau_{i 0}(t)$ are the unavoidable time-varying communication delays affecting the $i$-th agent when information is transmitted from its neighbor $j$ and from the leader respectively (in general $\tau_{i j}(t) \neq \tau_{j i}(t)$ ). The delay $\tau_{i j}(t)$ is bounded as $\tau_{i j}(t) \leq \tau$ [27], [28]; $\tau_{i j}(t)$ is known when the information is fed into the control algorithm, since each message is stamped with GPS-based time, whose precision is better than $100 \mathrm{~ns}$. The information relative to the predecessor is integrated with the same measures taken by on-board sensors (like radar, lidar, camera), thus improving the overall precision of measures. The effects of information loss will be analyzed in Sec. IV-C.

\section{Closed-loop Dynamics}

In this section we analytically prove the closed-loop stability of the platoon under the action of the consensus-based control. The proof of stability is based on the recast of the closed-loop dynamics as a set of functional differential equations for which it is possible to find a quadratic Lyapunov-Razumikhin function [24] and, hence, asymptotic stability is proven in the presence of heterogeneous time-varying communication delays.

To this goal, we define position and speed errors with respect to the reference signals $r_{0}(t), v_{0}(i=1, \ldots, N)$ as:

$$
\begin{aligned}
& \bar{r}_{i}=\left(r_{i}(t)-r_{0}(t)-h_{i 0} v_{0}-d_{i 0}^{s t}\right) ; \\
& \bar{v}_{i}=\left(v_{i}(t)-v_{0}\right) .
\end{aligned}
$$


Re-writing the coupling control action $u_{i}$ in terms of the state errors $\bar{r}_{i}$ and $\bar{v}_{i}$ and expressing headway constants $h_{i j}$ and standstill distances $d_{i j}^{s t}$ with respect to the leading vehicle, namely $h_{i j}=h_{i 0}-h_{j 0}$ and $d_{i j}^{s t}=d_{i 0}^{s t}-d_{j 0}^{s t}$, after some algebraic manipulation the closed-loop dynamics can be rewritten as $(i=1, \ldots, N)$ :

$$
\left\{\begin{array}{l}
\dot{\bar{r}}_{i}=\bar{v}_{i}, \\
M_{i} \dot{\bar{v}}_{i}=-\frac{1}{\Delta_{i}}\left(k_{i 0} a_{i 0}+\sum_{j=1}^{N} k_{i j} a_{i j}\right) \bar{r}_{i}-b \bar{v}_{i}(t)+ \\
\quad+\frac{1}{\Delta_{i}} \sum_{j=1}^{N} k_{i j} a_{i j}\left[\bar{r}_{j}\left(t-\tau_{i j}(t)\right)\right] .
\end{array}\right.
$$

To describe the platoon dynamics in presence of the timevarying delays associated to the different links in a more compact form we define the position and speed error vectors as $\bar{r}=\left[\bar{r}_{1}, \ldots, \bar{r}_{i} \ldots, \bar{r}_{N}\right]^{\top}, \bar{v}=\left[\bar{v}_{1}, \ldots, \bar{v}_{i} \ldots, \bar{v}_{N}\right]^{\top}$, and the error state vector as $\bar{x}(t)=\left[\bar{r}^{\top}(t) \bar{v}^{\top}(t)\right]^{\top}$. Moreover delays $\tau_{i j}$ in Eq. (9) can be recast as $\tau_{p}(t) \in\left\{\tau_{i j}(t): i, j=\right.$ $1,2, \ldots, N, i \neq j)\}$ for $p=1,2, \ldots, m$ with $m \leq N(N-1)$ $\left(0 \leq \tau_{p}(t) \leq \tau\right)$. Note that $m$ is equal to its maximum, $N(N-$ 1 ), if the platoon topology is represented by a directed complete graph and all time delays are different.

According to the above definitions, the closed loop platoon dynamics can be represented as the following set of functional differential equations:

$$
\dot{\bar{x}}(t)=A_{0} \bar{x}(t)+\sum_{p=1}^{m} A_{p} \bar{x}\left(t-\tau_{p}(t)\right),
$$

where $m$ is the total number of different time delays and

$$
A_{0}=\left[\begin{array}{cc}
0_{N \times N} & I_{N \times N} \\
-M \widetilde{K} & -M \widetilde{B}
\end{array}\right] \text { and } A_{p}=\left[\begin{array}{cc}
0_{N \times N} & 0_{N \times N} \\
M \widetilde{K}_{p} & 0_{N \times N}
\end{array}\right]
$$

being

$$
\begin{gathered}
M=\operatorname{diag}\left\{\frac{1}{M_{1}}, \ldots, \frac{1}{M_{N}}\right\} \in \mathbb{R}^{N \times N} ; \\
\widetilde{B}=\operatorname{diag}\{b, \ldots, b\} \in \mathbb{R}^{N \times N} ; \\
\widetilde{K}=\operatorname{diag}\left\{\tilde{k}_{11}, \ldots, \tilde{k}_{N N}\right\} \in \mathbb{R}^{N \times N}, \text { with } \tilde{k}_{i i}=\frac{1}{\Delta_{i}} \sum_{j=0}^{N} k_{i j} a_{i j} ;
\end{gathered}
$$

and $\tilde{K}_{p}=\left[\bar{k}_{p i j}\right] \in \mathbb{R}^{N \times N}(p=1, \ldots, m)$ the matrix defined according to the formalism adopted in [29] as:

$$
\bar{k}_{p i j}=\left\{\begin{array}{cc}
\frac{a_{i j} k_{i j}}{\Delta}, & j \neq i, \tau_{p}(\cdot)=\tau_{i j}(\cdot), \\
0, & j \neq i, \tau_{p}(\cdot) \neq \tau_{i j}(\cdot) . \\
0, & j=i .
\end{array}\right.
$$

\section{Stability Analysis}

From the Leibniz-Newton formula it is known that [30]:

$$
\bar{x}\left(t-\tau_{p}(t)\right)=\bar{x}(t)-\int_{-\tau_{p}(t)}^{0} \dot{\bar{x}}(t+s) d s .
$$

Hence, substituting Eq. (10) in Eq. (16) we have:

$$
\bar{x}\left(t-\tau_{p}(t)\right)=\bar{x}(t)-\sum_{q=0}^{m} A_{q} \int_{-\tau_{p}(t)}^{0} \bar{x}\left(t+s-\tau_{q}(t+s)\right) d s,
$$

where matrices $A_{0}, A_{1}, \ldots, A_{m}$ are defined in Eq. (11) and $\tau_{0}(t+s) \equiv 0$. Using the above transformation, the timedelayed model (Eq. (10)) can be transformed into:

$$
\begin{aligned}
& \dot{\bar{x}}(t)=A_{0} \bar{x}(t)+\sum_{p=1}^{m} A_{p} \bar{x}(t)+ \\
& -\sum_{p=1}^{m} \sum_{q=0}^{m} A_{p} A_{q} \int_{-\tau_{p}(t)}^{0} \bar{x}\left(t+s-\tau_{q}(t+s)\right) d s .
\end{aligned}
$$

From the definition in Eq. (11) it follows that $A_{p} A_{q}=0$ when $p=1, \ldots m$ and $q=1, \ldots, m$. Hence the system defined in Eq. (10) can be rewritten as:

$$
\dot{\bar{x}}(t)=F \bar{x}(t)-\sum_{p=1}^{m} C_{p} \int_{-\tau_{p}(t)}^{0} \bar{x}(t+s) d s
$$

where

$$
C_{p}=A_{p} A_{0}=\left[\begin{array}{cc}
0_{N \times N} & 0_{N \times N} \\
0_{N \times N} & M \widetilde{K}_{p}
\end{array}\right],
$$

and

$$
F=A_{0}+\sum_{p=1}^{m} A_{p}=\left[\begin{array}{ll}
0_{N \times N} & I_{N \times N} \\
-M \widehat{K} & -M \widetilde{B}
\end{array}\right]
$$

with

$$
\widehat{K}=-\sum_{p=1}^{m} \widetilde{K}_{p}+\widetilde{K} .
$$

Furthermore the following Lemmas hold:

Lemma 1. Supposing $k_{i}=\frac{k_{i 0} a_{i 0}}{\Delta_{i}} \geq 0(i=1, \ldots, N)$, the matrix $\widehat{K}$ in Eq. (22) is positive stable if and only if node 0 is globally reachable in $\overline{\mathcal{G}}$.

According to Lemma 1 the following matrix

$$
\widehat{K}_{M}=M \widehat{K}
$$

is also positive stable since $M>0$ (Eq. (12)).

Lemma 2. Let $F$ be the matrix defined in Eq. (21). F is Hurwitz stable if and only if $\widehat{K}_{M}$ (Eq. (23)) in Lemma 1 is positive stable and

$$
b>\max _{i}\left\{\frac{\left|\operatorname{Im}\left(\mu_{i}\right)\right|}{\sqrt{\operatorname{Re}\left(\mu_{i}\right)}} M_{i}\right\}
$$

being $\mu_{i}$ the $i$-th eigenvalue of $\widehat{K}_{M}(i=1, \ldots, N)$.

Lemmas 1 and 2 can be proved extending the proof in [22] to the case of closed-loop matrices depending from $m \leq N(N-1)$ time-varying delays. Platoon stability can be now proved as follows.

Theorem 2. Consider the system defined in Eq. (10) and take the control parameters in Eq. (7) as $k_{i j}>0$ and b such that

$$
b>b^{\star}=\max _{i}\left\{\frac{\left|\operatorname{Im}\left(\mu_{i}\right)\right|}{\sqrt{\operatorname{Re}\left(\mu_{i}\right)}} M_{i}\right\}
$$

where $\widehat{K}_{M}$ is defined in Eq. (23). Then, there exists a constant $\tau^{\star}>0$ such that, when $0 \leq \tau_{p}(t) \leq \tau<\tau^{\star}(p=1, \ldots, m)$,

$$
\lim _{t \rightarrow \infty} \bar{x}(t)=0 \text {, }
$$

if and only if node 0 is globally reachable in $\overline{\mathcal{G}}$. 
Proof. (Sufficiency). Since node 0 is globally reachable in $\overline{\mathcal{G}}$, from Lemma 1 it follows that the matrix $\widehat{K}_{M}$ is positive stable. Setting $b$ as in Eq. (25), the hypothesis of Lemma 2 is satisfied, hence the matrix $F$ defined in Eq. (21) is Hurwitz stable and from Lyapunov theorem there exists a positive definite matrix $P \in \mathbb{R}^{2 N \times 2 N}$ such that

$$
P F+F^{\top} P=-Q ; \quad Q=Q^{\top}>0 .
$$

Consider the following Lyapunov-Razumikhin candidate function (i.e., satisfying condition of Lyapunov-Razumikin Theorem 1)

$$
V(\bar{x})=\bar{x}^{\top} P \bar{x} .
$$

From Eq. (19), taking the derivative of $V$ along Eq. (10) gives

$$
\dot{V}(\bar{x})=\bar{x}^{\top}\left(P F+F^{\top} P\right) \bar{x}-\sum_{p=1}^{m} 2 \bar{x}^{\top} P C_{p} \int_{-\tau_{p}(t)}^{0} \bar{x}(t+s) d s .
$$

Now for any positive definite matrix $\Xi$ it is possible to show that $2 a^{\top} c \leq a^{\top} \Xi a+c^{\top} \Xi^{-1} c$ according to [23]. Therefore, setting $a^{\top}=-\bar{x}^{\top} P C_{p}, c=\bar{x}(t+s), \Xi=P^{-1}$, and integrating both sides of the inequality, we can write

$$
\begin{aligned}
\dot{V}(\bar{x}) \leq & \bar{x}^{\top}\left(P F+F^{\top} P\right) \bar{x}+\sum_{p=1}^{m}\left[\tau_{p}(t) \bar{x}^{\top} P C_{p} P^{-1} C_{P}^{\top} P \bar{x}+\right. \\
& \left.+\int_{-\tau_{p}(t)}^{0} \bar{x}^{\top}(t+s) P \bar{x}(t+s) d s\right] .
\end{aligned}
$$

According to the hypotheses of the Lyapunov-Razumikin Theorem [24], choose now the following continuous non decreasing function $\psi_{4}(s)=q s$ (for some constant $q>1$ ) and the continuous, non negative, non decreasing function $\psi_{3}(s)=\left(\lambda_{\min }(Q)-\tau \lambda_{\max }(H)\right) s^{2}$; being $\lambda_{\min }(Q)$ the minimum eigenvalue of $Q ; \lambda_{\max }(H)$ the maximum eigenvalue of the matrix $H$ defined as $H=\sum_{p=1}^{m} P C_{p} P^{-1} C_{P}^{\top} P+q P$;

$$
\tau<\tau^{\star}=\frac{\lambda_{\min }(Q)}{\lambda_{\max }(H)} .
$$

After some simple algebraic manipulations, when

$$
V(\bar{x}(t+\theta))<\psi_{4}(V(\bar{x}))=q V(\bar{x}(t)),-\tau \leq \theta \leq 0,
$$

Eq. (30) becomes

$$
\dot{V}(\bar{x}) \leq-\left(\lambda_{\min }(Q)-\tau \lambda_{\max }(H)\right)\|\bar{x}\|^{2}=-\psi_{3}(\|\bar{x}\|) .
$$

In so doing, the sufficient condition is proven.

(Necessity). Eq. (10) is asymptotically stable for any time delay $\tau_{p}(t)<\tau^{\star}, p=1, \ldots, m$. Letting $\tau_{p}(t) \equiv 0(p=1, \ldots, m)$ in Eq. (10), it follows from Eq. (19) that system $\dot{\bar{x}}=F \bar{x}$ with $\mathrm{F}$ defined in Eq. (21) is asymptotically stable. As all the eigenvalues of $\mathrm{F}$ have negative real parts, Lemma 2 implies that $\widehat{K}_{M}$ is positive stable. Now applying Lemma 1 the theorem is proven.

Control gains are set inside the consensus region to analyti-

\begin{tabular}{|c|c|}
\hline Parameter & Value \\
\hline \multicolumn{2}{|r|}{ Bernoullian channel } \\
\hline PER $p$ & $0.3,0.5$ and 0.6 \\
\hline \multicolumn{2}{|r|}{ Gilbert-Elliott channel } \\
\hline PER $p$ (GOOD) & 0.2 \\
\hline PER $p$ (BAD) & $\begin{array}{l}0.7 \\
\end{array}$ \\
\hline state duration & $\sim \exp \left(0.5 \mathrm{~s}^{-1}\right)(\mathbb{E}[X]=2 \mathrm{~s})$ \\
\hline \multicolumn{2}{|r|}{ Realistic channel } \\
\hline Path loss model & Free space $(\alpha=2.0)$ \\
\hline Fading model & Nakagami-m $(m=3)$ \\
\hline PHY/MAC model & IEEE $802.11 \mathrm{p} / 1609.4$ single channel $(\mathrm{CCH})$ \\
\hline Frequency & $5.89 \mathrm{GHz}$ \\
\hline Bitrate & $6 \mathrm{Mbits}^{-1}(\mathrm{QPSK} \mathrm{R}=1 / 2)$ \\
\hline Access category & AC_VI \\
\hline MSDU size & 200 B (byte) \\
\hline Transmit power & $20 \mathrm{dBm}$ \\
\hline Beacon frequency & $10 \mathrm{~Hz}$ \\
\hline
\end{tabular}
cally guarantee disturbance attenuation for all frequencies of interest (i.e., string stability with respect to disturbances acting on the leader motion). As common practice, this has been

\begin{tabular}{|c|c|}
\hline Freeway length & $10 \mathrm{~km}$ \\
\hline Lanes & 4 (two-way) \\
\hline Cars percentage (length $4 \mathrm{~m}$ ) & $50 \%$ \\
\hline Trucks percentage (length $20 \mathrm{~m}$ ) & $20 \%$ \\
\hline Vans percentage (length $5 \mathrm{~m}$ ) & $30 \%$ \\
\hline Inter-vehicle time & $\sim \exp \left(0.7276 \mathrm{~s}^{-1}\right)(\mathbb{E}[X]=1.374 \mathrm{~s}[31]$ \\
\hline Cars'speed & $\sim U\left(100 \mathrm{~km} \mathrm{~h}^{-1}, 160 \mathrm{~km} \mathrm{~h}^{-1}\right)$ \\
\hline Trucks' speed & $80 \mathrm{~km} \mathrm{~h}^{-1}$ \\
\hline Vans' speed & $100 \mathrm{~km} \mathrm{~h}^{-1}$ \\
\hline Platoon size & 8 and 16 cars \\
\hline Platooning car max acceleration & $2.3 \mathrm{~m} \mathrm{~s}^{-2}$ \\
\hline Platooning car mass & $1460 \mathrm{~kg}$ \\
\hline Platooning car length $l_{i}$ & $4 \mathrm{~m}$ \\
\hline Headway time $h_{i j}$ & $0.8 \mathrm{~s}$ \\
\hline Control gains $k_{i j}$ & $\begin{array}{r}k_{10}=460, k_{i 0}=80(i \neq 0, i \neq 1) \\
k_{i j}=860, k_{i j}=0 \text { otherwise }\end{array}$ \\
\hline Control gains $b_{i}$ & $\kappa_{i, i-1}-000, \kappa_{i j}-1800$ \\
\hline Distance at standstill $d^{s t}$ & $15 \mathrm{~m}$ \\
\hline Freeway fill-up $t$ & $500 \mathrm{~s}$ \\
\hline Network warm-up time & $10 \mathrm{~s}$ \\
\hline Data recording time & $50 \mathrm{~s}$ \\
\hline
\end{tabular}
analytically achieved for our control algorithm by enclosing all the time-varying delays within a unique upper bound and then
Table I

NETWORK SIMULATION PARAMETERS.

Table II

TRAFFIC SIMULATION PARAMETERS FOR THE REALISTIC SCENARIO.

deriving in the Laplace domain the complementary sensitivity functions, exploiting a first-order Padé approximation for the delay. In so doing $k_{i j}$ and $b_{i}$ guarantee both consensus and string stability (values are reported in Tab. II).

\section{EXPERIMENTAL ANALYSIS}

\section{A. Network and Traffic Scenario}

We use the PLEXE simulator described in [32], based on Veins [5], where the traditional CACC proposed in [3] is already available, and the actuation lag (i.e., the delay between the control decision and its actual realization in the vehicle due to inertial and mechanical limits) is correctly modeled. It permits the investigation of platooning systems by coupling realistic vehicle dynamics with realistic wireless network simulation. Eq. (7) is implemented in the simulator as platoon control system, properly distributed in each vehicle. The simulation code is available to the community through the Veins site.

Regarding the channel models, we first consider two simple setups to explore basic convergence and stability properties of 


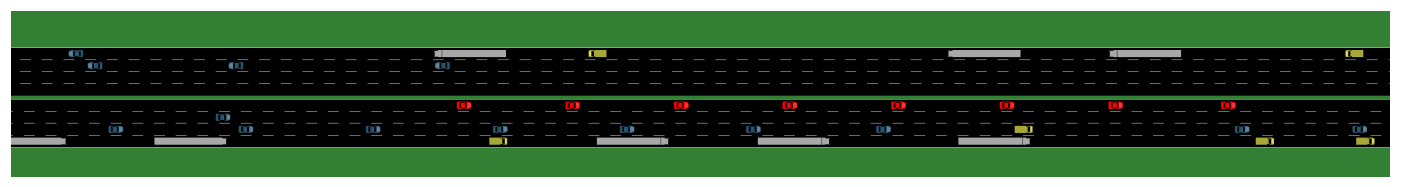

Figure 1. Screenshot of the realistic scenario. Human-driven vehicles in white, blue, and yellow, and platooning cars in red on the left-most lane.

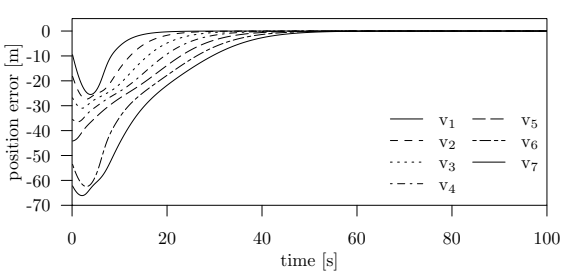

(a)

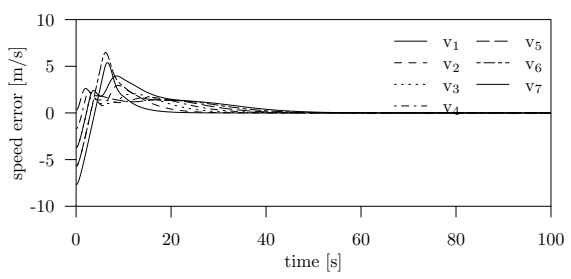

(b)

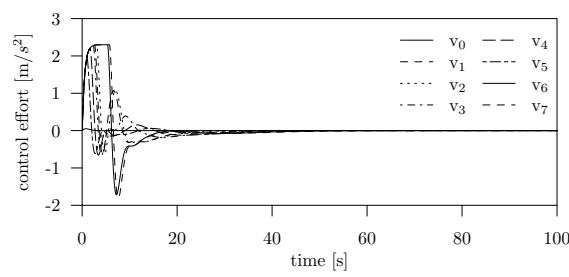

(c)

Figure 2. Basic convergence analysis with $v_{0}=100 \mathrm{~km} \mathrm{~h}^{-1}, N+1=8$ vehicles. Platoon creation and maintenance: (a) time history of the position errors computed as $r_{i}(t)-r_{0}(t)-h_{i 0} v_{0}-d_{i 0}^{s t}$; (b) time history of the vehicles speed error with respect to the leader computed as $v_{i}(t)-v_{0}$; (c) time history of the control effort in $\mathrm{ms}^{-2}$.

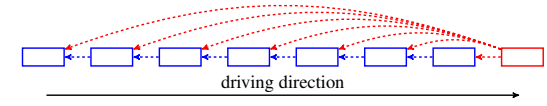

Figure 3. Vehicular topology in the simulation scenario.

the system. In particular, we first use a Bernoullian channel, i.e., with independent random losses and different Packet Error Rates (PERs), and then we employ a Gilbert-Elliott channel driven by a two-state Markov chain. Each state represents the current channel status, which can be either in good or in bad conditions. The channel conditions determine the PER to be used, enabling the possibility to simulate burst errors. State durations are drawn from an exponential distribution. The third network scenario we take into account is the most realistic: we consider a $10 \mathrm{~km}$ freeway where human-driven vehicles travel on the road generating wireless interferences. As channel model, we employ a free-space path loss coupled with Nakagami-m fading. We use a fully fledged IEEE $802.11 \mathrm{p} / 1609.4$ model configured with typical parameters, and consider a beacon frequency of $10 \mathrm{~Hz}$, both for automated and human-driven vehicles. Concerning the road traffic simulation we consider different kind of vehicles traveling in both directions. The simulation includes cars, vans, and trucks with different percentages and speeds, which are injected with an exponentially distributed inter-vehicle time. At simulation time $500 \mathrm{~s}$ the platoon is injected in the middle of the freeway and communications are enabled. After a warm-up time of $10 \mathrm{~s}$ we start to record motion data about vehicles in the platoon. Tabs. I and II summarize all relevant parameters for both network and traffic simulation.

To show the stability and robustness of the proposed control strategy an experimental analysis has been performed involving different driving leader maneuvers, in particular: (i) Consensus: starting from different initial conditions, the platoon has to reach and than maintain the reference behavior as imposed by the leader according to the desired spacing policy; (ii) Leader tracking: followers have to correctly track the timevarying leader speed, $v_{0}$; (iii) Sinusoidal: a periodic disturbance is acting on the leader motion. Note that fluctuations have to be attenuated toward the tail of the platoon. The chosen control topology is the one considered in [33] and coherent with [3], where the leader communicates with all the vehicles in broadcast, and every vehicle shares information with its follower (see Fig. 3). Fig. 1 shows a screenshot of the simulation. We remark that the algorithm convergence is not restricted to the case of classical predecessor-following architecture based on pairwise interactions [2], but it ensures platoon stability for all those topologies that satisfy hypotheses of Theorem 2. Results are illustrated referring to growing complexity in network load and traffic scenario. Moreover, a brief comparison with a classical CACC [3] control technique has been carried out.

\section{B. Basic Convergence Analysis}

In this section we refer to the case study of a platoon composed of 7 vehicles plus a leader. No packets are ever lost in this first scenario. Control parameters are tuned inside the consensus region according to Theorem 2 to achieve acceptable transient performance and to guarantee string stability. The selected control parameters are reported in Tab. II. Figs. 2a and $2 \mathrm{~b}$ show the results for the consensus scenario. The results confirm the ability of the proposed approach of creating and maintaining the platoon. All vehicles - starting from distances different from the one required by the spacing policy - reach the consensus and converge toward the desired positions and the leader speed, despite the presence of network delays during the information exchange. Furthermore, according to the theoretical derivation, the control effort reduces to zero once the control goal is achieved, as depicted in Fig. 2c. The consensus is theoretically guaranteed for a constant leader speed, but the controller stability leaves ample control margins to ensure that the platoon is able to track the leader. We test the ability of the proposed strategy of tracking the leader when it accelerates from $0 \mathrm{~km} \mathrm{~h}^{-1}$ to $90 \mathrm{~km} \mathrm{~h}^{-1}$ (with a constant acceleration of $0.5 \mathrm{~m} \mathrm{~s}^{-2}$ ). Results in Fig. 4 show that the approach is able to achieve tracking by bringing all vehicles to the required speed 


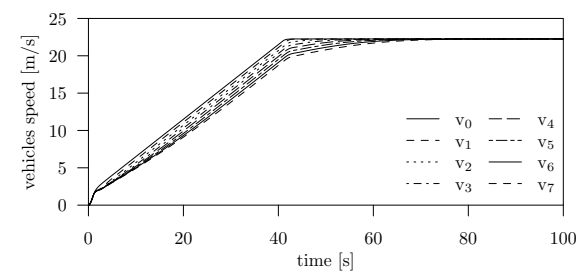

Figure 4. Leader tracking maneuver: time history of the vehicles speed.

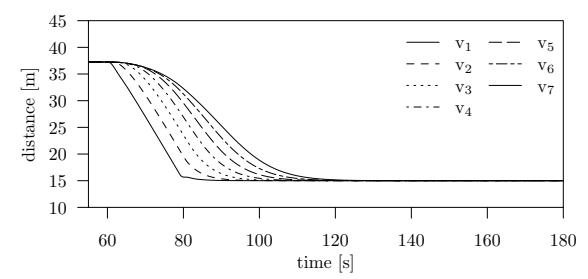

Figure 5. Braking maneuver: time history of bumper to bumper distances computed as $r_{i-1}(t)-r_{i}(t)-l_{i-1}$.

and mutual positions (not shown).

To confirm the tracking performance of our algorithm, we test the controller in a braking scenario. Results in Fig. 5 show how the platoon reacts in the case of a braking maneuver performed by the leader from $100 \mathrm{~km} \mathrm{~h}^{-1}$ to a full stop. The platoon maintains the secure inter-vehicular distance, avoids collisions, and converges to stand-still distances at rest.

We dedicate further experiments to investigate if and how speed and acceleration fluctuations are attenuated downstream the string of vehicles of the platoon (string stability) when a periodic disturbance is acting on leader's speed. Results in Fig. 6, referring to a sinusoidal disturbance

$$
\delta(t)=A \cos \left(\frac{6}{100} \pi \mathrm{t}\right), \mathrm{A}=2.7 \mathrm{~m} \mathrm{~s}^{-1},
$$

confirm the string stable behavior of the platoon. The position error of vehicles with respect to its predecessor shows that the sinusoidal disturbance is attenuated downstream the string of vehicles.

As final test, we check the convergence for a platoon of 16 vehicles. The platoon still reaches the consensus conditions (Figs. 7a and 7b) and shows a string stable behavior (Fig. 7c). Moreover in this scenario we have re-tuned the controller to ensure a constant and very small $(5 \mathrm{~m})$ bumper to bumper distance and not a constant time headway.

\section{Simulations using Packet Losses}

In this scenario we analyze the performance of the proposed control approach in a more realistic scenario that considers Bernoulli and Gilbert-Elliott packet losses parameterized as in Tab. I. Concerning the Bernoullian channel (graphical results are not shown for the sake of brevity), we verified that the consensus is well guaranteed in the case of a PER up to $60 \%$. Performance start to slightly deteriorate around $60 \%$ PER, but the platoon motion is still preserved and both position and velocity errors still converge to zero. Consensus is lost for packet loss probabilities above $70 \%$. Regarding the GilbertElliott channel (see parameters in Tab. I), Figs. 8a and 8b show position and speed errors as function of time, proving

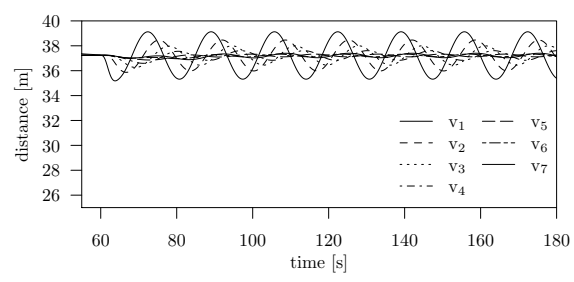

Figure 6. Robustness with respect to the sinusoidal disturbance (Eq. (34)) acting on the leader speed: time history of bumper to bumper distances computed as $r_{i-1}(t)-r_{i}(t)-l_{i-1}$.

that consensus can be reached in this setup as well. All these results show very high resilience to packet loss, which may appear surprising. The explanation lies in the high sampling rate of $10 \mathrm{~Hz}$ beaconing compared to the system dynamics given by Eqs. (4) to (6), which, given the vehicles masses, are much slower. Thus even the loss of a large fraction of messages has just the effect of a mild under sampling of the system compared to the default one, which is however much higher than the minimum required. Finally, we highlight the relationship between packet loss and $\tau_{i j}(t)$. Recall that $\tau_{i j}(t)$ are measured based on GPS timestamps in the messages, hence when a message is lost, the algorithms uses the last available information, thus $\tau_{i j}(t)$ actually "jumps," increasing of a beacon interval, just to return to a smaller value when the next valid message is received. Thus the resilience to message loss, also implies the robustness to the variable $\tau_{i j}(t)$ studied in the theoretical part.

\section{Simulations in High Density Traffic Scenario}

In the realistic freeway scenario described in Sec. IV-A, we simulate the consensus, the leader tracking, and the sinusoidal disturbance, but for the sake of brevity we report the results of the tracking and the sinusoidal ones only. Fig. 9 shows the speed profiles as function of time for the vehicles in the platoon for the leader tracking scenario. The leader accelerates from $80 \mathrm{~km} \mathrm{~h}^{-1}$ to $130 \mathrm{~km} \mathrm{~h}^{-1}$ with a constant acceleration of $1.5 \mathrm{~m} \mathrm{~s}^{-2}$. Despite the interferences caused by other vehicles, all cars in the platoon correctly track the leader's maneuver, and the differences with Fig. 4 are minor. In the second scenario, the leader accelerates and decelerates in a sinusoidal fashion around the average speed of $110 \mathrm{~km} \mathrm{~h}^{-1}$ with a frequency of $0.2 \mathrm{~Hz}$. In Fig. 10 we plot the bumper-to-bumper distance for all the cars in the platoon. As in Fig. 6, the controller successfully maintains string-stability by attenuating the error along the platoon. Indeed, the oscillation is barely noticeable already at vehicle number 3 . Nevertheless, there are minor imperfections caused by packet losses. For example, between simulation times $602 \mathrm{~s}$ and $606 \mathrm{~s}$, it can be noticed that vehicle 7 looses its reference position. The error is however in the order of $20 \mathrm{~cm}$, thus the system can still be considered safe and robust.

\section{E. A Brief Comparison with a traditional controller}

We compare here the performance of our approach with the CACC controller illustrated in [3, Chapter 7] as implemented 


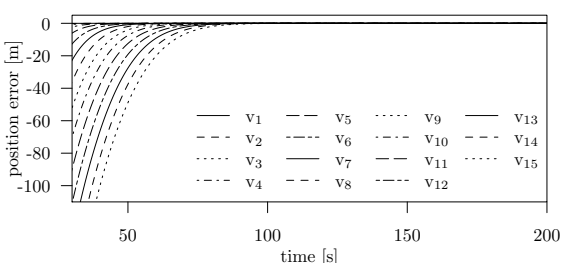

(a)

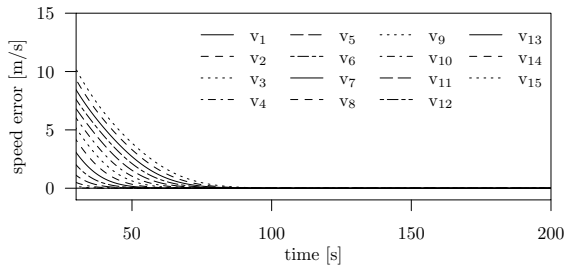

(b)

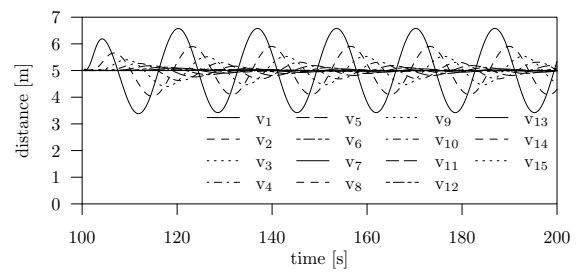

(c)

Figure 7. Platoon of $N+1=16$ vehicles. (a) Platoon creation and maintenance: time history of the position errors computed as $r_{i}(t)-r_{0}(t)-h_{i 0} v_{0}-d_{i 0}^{s t}$; (b) time history of the vehicles' speed error with respect to the leader computed as $v_{i}(t)-v_{0}$. (c) Robustness with respect to the sinusoidal disturbance (Eq. (34)) acting on the leader speed: time history of bumper to bumper distance computed as $r_{i-1}(t)-r_{i}(t)-l_{i-1}$.

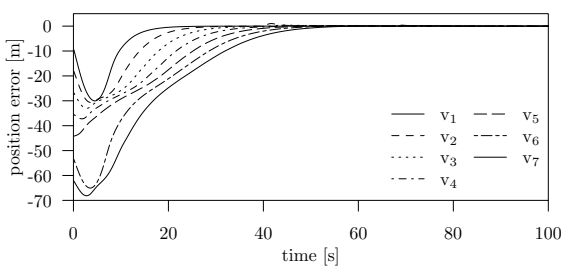

(a)

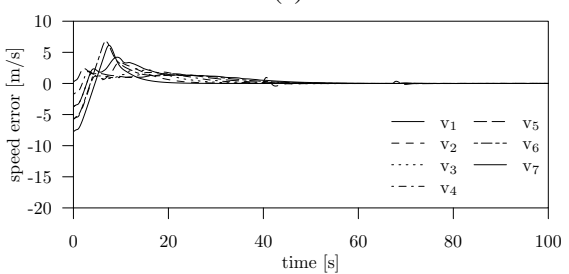

(b)

Figure 8. Consensus in presence of packet losses. Gilbert-Elliott transmission channel: (a) position errors computed as $r_{i}(t)-r_{0}(t)-h_{i 0} v_{0}-d_{i 0}^{s t}$; (b) speed errors computed as $v_{i}(t)-v_{0}$.

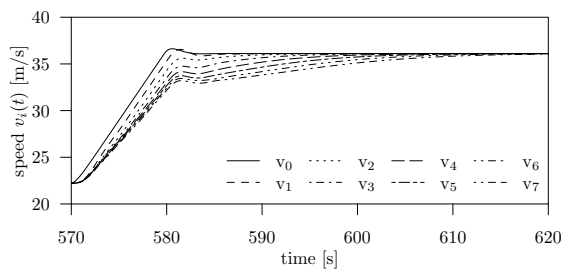

Figure 9. Leader tracking maneuver in the realistic network scenario: time history of vehicles' speed.

by Eqs. (7)-(12) in [32], considered one of the most performing controllers able to stabilize a platoon with an inter-vehicle distance independent from the platoon speed. In this section, for sake of clarity, we refer to this CACC algorithm as rajc and compare it with our Consensus-based Control (cbc).

First of all, our proposal allows changing the values assumed by the headway time constant without requiring a specific tuning of the control parameters. Hence, different headway time values $h_{i j}$ can be used both at different speeds, but also within the same platoon for different cars allowing, for instance, increased safety in presence of heterogeneous vehicles without compromising the ensemble behavior. Control flexibility is increased [34], but also convergence time is faster. Results in Fig. 11 show how the convergence time (i.e., the time $\tau_{5 \%}$ and $\tau_{1 \%}$ necessary to reach the desired platoon configuration with an error smaller than $5 \%$ or $1 \%$, respectively) varies as a function of $h_{i j}$ for cbc and rajc in a platoon forming at

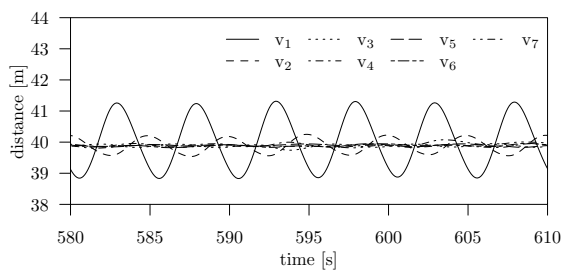

Figure 10. Sinusoidal disturbance on leader motion in the realistic network scenario: bumper to bumper distances computed as $r_{i-1}(t)-r_{i}(t)-l_{i-1}$.

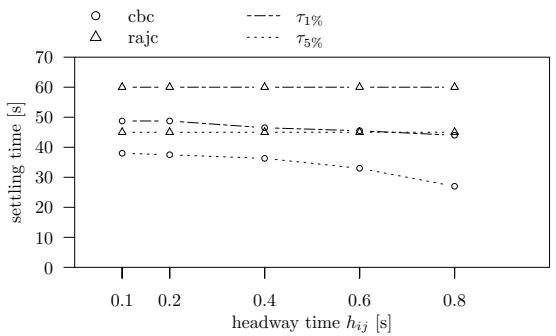

Figure 11. Platoon convergence time $\tau$ vs. headway-time $h_{i j}$ for Consensus based Control (cbc) and the traditional rajc [3, Chapter 7] CACC.

$100 \mathrm{~km} \mathrm{~h}^{-1}$. Convergence times are measured starting from the instant the leader announces the platoon formation. The other cars join the platoon every $2 \mathrm{~s}$, and immediately start following the control algorithm. The convergence time of cbc is about $20 \%$ faster and decreases with $h_{i j}$.

Convergence is very important, but noise rejection and fluctuations damping are just as important, both for safety and for driving comfort. We evaluate this property imparting a sinusoidal speed "noise" of different frequencies to the leader, and measuring the ratio $\frac{\left\|a_{i}(t)\right\|_{\infty}}{\left\|a_{0}(t)\right\|_{\infty}}$, of all the following vehicles accelerations compared to the leader. The leader speed oscillation amplitude is $\pm 1.4 \mathrm{~km} \mathrm{~h}^{-1}$. A good platoon control system should increase the damping as the frequency of this oscillation increases, because as the frequency increases they are more and more perceived as vibrations, and hence are annoying, while the inter-vehicle distance does not change much, so stabilizing the speed of the followers does not hamper safety. Results in Fig. 12 confirm that cbc damping increases with frequency and for high frequencies is already very good also for the first vehicle, while as the frequency decreases then the damping converges to 1 (no damping), as these low frequency fluctuations may correspond to real changes of the leader speed and not to noise. rajc damping is instead marginally smaller than 1 even for frequencies as high as $1 \mathrm{~Hz}$ that for a car speed are really violent vibrations and nothing else. 


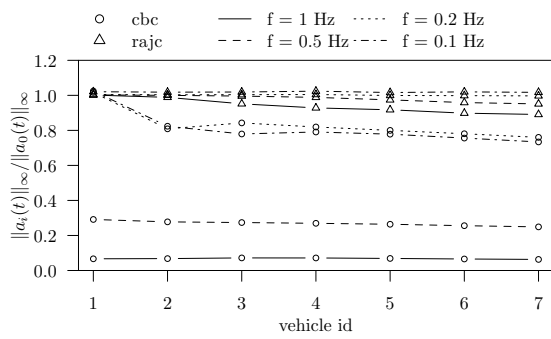

Figure 12. Damping of the magnitude of the leader speed oscillations for different frequencies for cbc and rajc.

\section{CONCLUSiOnS}

In this paper we have proposed a novel consensus-based control approach for vehicle platoons that natively include in the design the communications' delays and the topology of the agents network that implements the consensus algorithm, which thus becomes a design decision. We have analytically proven the stability and convergence of the platooning algorithm in presence of time-variable heterogeneous delays. The resulting protocol has been implemented in Veins on top of a standard DSRC/WAVE communication infrastructure and it has been evaluated in several realistic scenarios. Finally it has been compared with a classic CACC approach well known in literature. Future work will be devoted to extending the analysis to dynamically changing topologies, which can improve the feasibility of the complex driving maneuvers (overtaking, merging/splitting platoons, etc.) that are needed to implement fully autonomous cooperative driving. Furthermore, the theoretical analysis will be extended to understand the limits of consensus-based platoons control in dynamic conditions, as well as in the extremely compact platoons that can reduce fuel consumption thanks to reduced air drag.

\section{REFERENCES}

[1] S. Shladover, C. Desoer, J. Hedrick, M. Tomizuka, J. Walrand, W.-B Zhang, D. McMahon, H. Peng, S. Sheikholeslam, and N. McKeown, "Automated Vehicle Control Developments in the PATH Program," IEEE Trans. on Vehicular Technology, vol. 40, no. 1, pp. 114-130, Feb 1991.

[2] J. Ploeg, B. Scheepers, E. van Nunen, N. van de Wouw, and H. Nijmeijer, "Design and Experimental Evaluation of Cooperative Adaptive Cruise Control," in IEEE ITSC 2011, Washington, DC, USA, Oct 2011, pp 260-265.

[3] R. Rajamani, Vehicle Dynamics and Control, 2nd ed. Springer, 2012.

[4] L. Wang, G. Yin, H. Zhang, L. Xu, A. Syed, G. Yin, A. Pandya and H. Zhang, "Control of Vehicle Platoons for Highway Safety and Efficient Utility: Consensus with Communications and Vehicle Dynamics," Springer Jou. of Systems Science and Complexity, vol. 27, no. 4, pp 605-631, Aug 2014.

[5] C. Sommer, R. German, and F. Dressler, "Bidirectionally Coupled Network and Road Traffic Simulation for Improved IVC Analysis," IEEE Trans. on Mobile Computing, vol. 10, no. 1, pp. 3-15, Jan 2011.

[6] "Trial-Use Standard for Wireless Access in Vehicular Environments (WAVE) - Multi-channel Operation,” IEEE, Std 1609.4, Feb 2011.

[7] "Wireless Access in Vehicular Environments," IEEE, Std 802.11p-2010, Jul 2010.

[8] M. Segata and R. Lo Cigno, "Automatic Emergency Braking: Realistic Analysis of Car Dynamics and Network Performance," IEEE Trans. on Vehicular Technology, vol. 62, no. 9, pp. 4150-4161, Oct 2013.

[9] H. Hao and P. Barooah, "Stability and Robustness of Large Platoons of Vehicles with Double-integrator Models and Nearest Neighbor Interaction," Wiley Int. Jou. of Robust and Nonlinear Control, vol. 23 no. 18, pp. 2097-2122, Dec 2013.

[10] K. Santhanakrishnan and R. Rajamani, "On Spacing Policies for Highway Vehicle Automation," IEEE Trans. on Intelligent Transportation Systems, vol. 4, no. 4, pp. 198-204, Dec 2003.
[11] D. Swaroop and J. Hedrick, "String Stability of Interconnected Systems," IEEE Trans. on Aut. Control, vol. 41, no. 3, pp. 349-357, Mar 1996.

[12] X. Liu, A. Goldsmith, S. Mahal, and J. Hedrick, "Effects of Communication Delay on String Stability in Vehicle Platoons," in IEEE ITSC, Oakland, CA, USA, Aug 2001, pp. 625-630.

[13] P. Fernandes and U. Nunes, "Platooning with IVC-enabled Autonomous Vehicles: Strategies to Mitigate Communication Delays, Improve Safety and Traffic Flow," IEEE Trans. on Intelligent Transportation Systems, vol. 13, pp. 91-106, Mar 2012.

[14] _ "Platooning of Autonomous Vehicles with Intervehicle Communications in SUMO Traffic Simulator," in IEEE ITSC, Madeira, Portugal, Sept 2010, pp. 1313-1318.

[15] C. Lei, E. van Eenennaam, W. Wolterink, G. Karagiannis, G. Heijenk, and J. Ploeg, "Impact of Packet Loss on CACC String Stability Performance," in IEEE ITST, St. Petersburg, Russia, Aug 2011, pp. 381-386.

[16] G. Naus, R. Vugts, J. Ploeg, M. van de Molengraft, and M. Steinbuch, "String-Stable CACC Design and Experimental Validation: A FrequencyDomain Approach," IEEE Trans. on Vehicular Technology, vol. 59, no. 9 , pp. 4268-4279, Nov 2010.

[17] R. Olfati-Saber, J. A. Fax, and R. Murray, "Consensus and Cooperation in Networked Multi-Agent System," in Proc. of the IEEE, vol. 95, no. 1, Jan 2007 , pp. 215-233.

[18] R. Szalai and G. Orosz, "Decomposing the Dynamics of Heterogeneous Delayed Networks with Applications to Connected Vehicle Systems," APS Phys. Rev. E, vol. 88, no. 4, pp. 902-906, Oct 2013.

[19] V. Milanés, S. Shladover, J. Spring, C. Nowakowski, H. Kawazoe, and M. Nakamura, "Cooperative Adaptive Cruise Control in Real Traffic Situations," IEEE Trans. on Intelligent Transportation Systems, vol. 15, no. 1, pp. 296-305, Feb 2014.

[20] K. Karlsson, C. Bergenhem, and E. Hedin, "Field Measurements of IEEE 802.11p Communication in NLOS Environments for a Platooning Application," in IEEE VTC2012-Fall, Quebec City, QC, Canada, Sept 2012, pp. $1-5$.

[21] M. Segata, B. Bloessl, S. Joerer, C. Sommer, R. Lo Cigno, and F. Dressler, "Vehicle Shadowing Distribution Depends on Vehicle Type: Results of an Experimental Study," in IEEE VNC 2013, Boston, MA, USA, Dec 2013, pp. 242-245.

[22] M. di Bernardo, A. Salvi, and S. Santini, "Distributed consensus strategy for platooning of vehicles in the presence of time-varying heterogeneous communication delays," IEEE Trnas. on Intelligent Transportation Systems, vol. PP, no. 99, pp. 1-11, Sepr. 2014.

[23] R. A. Horn and C. R. Johnson, Matrix Analisis. Cambridge Univ. Press, 1987.

[24] J. K. Hale and S. M. V. Lunel, Introduction to Functional Differential Equations. Springer-Verlag, 1993.

[25] E. Coelingh and S. Solyom, "All Aboard the Robotic Road Train," IEEE Spectrum, vol. 49, no. 11, pp. 34-39, Nov 2012.

[26] S. Darbha and K. Rajagopal, "Intelligent Cruise Control Systems and Traffic Flow Stability," Elsevier Transp. Research Part C: Emerging Technologies, vol. 7, no. 6, pp. 329-352, Dec 1999.

[27] A. Botta, A. Pescapè, and G. Ventre, "Quality of Service Statistics over Heterogeneous Networks: Analysis and Applications," Esevier European Jou. of Op. Research, vol. 191, no. 3, pp. 1075-1088, Dec 2008.

[28] R. P. Karrer, I. Matyasovszki, A. Botta, and A. Pescapé, "Experimental Evaluation and Characterization of the Magnets Wireless Backbone," in ACM WiNTECHO6, Los Angeles, CA, USA, Sept. 2006, pp. 26-33.

[29] W. Yang, A. Bertozzi, and X. Wang, "Stability of a Second Order Consensus Algorithm with Time Delay," in IEEE CDC, Cancun, Mexico, Dec 2008, pp. 2926-2931.

[30] K. R. Stromberg, An Introduction to Classical Real Analysis. Springer, 1981.

[31] N. Wisitpongphan, F. Bai, P. Mudalige, and O. K. Tonguz, "On the Routing Problem in Disconnected Vehicular Ad-hoc Networks," in IEEE INFOCOM 2007, Anchorage, AK, USA, May 2007, pp. 2291-2295.

[32] M. Segata, S. Joerer, B. Bloessl, C. Sommer, F. Dressler, and R. Lo Cigno, "PLEXE: A Platooning Extension for Veins," in IEEE VNC, Paderborn, Germany, Dec 2014.

[33] T. Murray, M. Cojocari, and H. Fu, "Measuring the Performance of IEEE 802.11p Using NS-2 Simulator for Vehicular Networks," in IEEE EIT, Ames, IA, USA, May 2008, pp. 498-503.

[34] S. Oncü, N. van de Wouw, and H. Nijmeijer, "Cooperative Adaptive Cruise Control: Tradeoffs Between Control and Network Specifications," in IEEE ITSC 2011, Washington, DC, USA, Oct 2011, pp. 2051-2056. 